\title{
Molecular Polarity and the Structure of Liquid Methanol
}

\author{
G. Pálinkás*, Y. Tamura, E. Spohr, and K. Heinzinger \\ Max-Planck-Institut für Chemie (Otto-Hahn-Institut), Mainz, Federal Republic of Germany
}

Z. Naturforsch. 43a, 43-46 (1988); received October 8, 1987

The structure of liquid methanol as a function of the molecular polarity has been studied by MD simulations. The results obtained by scaling the partial Coulombic charges located at the molecular interaction sites show that the polar forces leading to the hydrogen bonding phenomena also influence the relative orientation of nearest neighbor molecules and thus their local packing. It is shown that hydrogen bonding in liquid methanol contributes considerably to the X-ray structure function. The changes with polarity of the pair interaction energy distributions, the intramolecular geometry, and the vibrational frequencies are reported. The results are compared with those obtained from RISM calculations.

\section{Introduction}

The structure of a hydrogen bonded liquid is determined by the polar orientational forces responsible for hydrogen bonding as well as by the packing requirements due to the shape of the molecules. The influence of polarity on the structure of liquid water and methanol has already been studied by the extended RISM integral equation theory $[1,2]$. The calculations based on the MCY model for water [3] and the TIPS model for methanol [4] have demonstrated the ability of the RISM theory to provide a qualitative description of the hydrogen bonding phenomena in both liquids. The results obtained as a function of molecular polarity - by scaling the partial Coulombic charges $q_{\alpha}^{\prime}=\lambda q_{\alpha}$ located at the molecular interaction sites indicate that many features of the water and methanol structure are dominated by packing effects and are insensitive to the presence of polar interactions.

For liquid methanol RISM calculations [2] and Monte Carlo simulations [4] have been performed with the same interaction potential and $\lambda=1$. Qualitative agreement has been found for all site-site radial distribution functions (RDF) except the $\mathrm{O}-\mathrm{O}$ one. The RISM method considerably underestimates the number of $\mathrm{O}-\mathrm{O}$ pairs at short distances. With decreasing polarity the RISM calculations show dramatic changes for the $\mathrm{O}-\mathrm{H}$ and $\mathrm{H}-\mathrm{H}$ RDFs while

\footnotetext{
* Permanent address: Central Research Institute for Chemistry, Hungarian Academy of Sciences, Budapest, Hungary.

Reprint requests to Dr. K. Heinzinger, Max-Planck-Institut für Chemie, P.O.B 3060, D-6500 Mainz.
}

they are moderate for the $\mathrm{H}-\mathrm{CH}_{3}$ and $\mathrm{O}-\mathrm{O}$ and negligible for the $\mathrm{O}-\mathrm{CH}_{3}$ and $\mathrm{CH}_{3}-\mathrm{CH}_{3}$ ones. Consequently the $\mathrm{X}$-ray structure function does practically not change with polarity, which has led to the conclusion that hydrogen bonding in liquid methanol gives only minor contributions to the $\mathrm{X}$-ray scattering pattern [2]. Computer simulations for liquid methanol with different polarities have not been reported so far.

In the present work MD simulations have been performed with a newly developed flexible three site model for methanol [5] and three different scaling factors $\lambda$. The calculated site-site RDFs and the X-ray structure function are compared with the results of the RISM calculations. The effect of decreasing polarity on the pair interaction energy distribution, the intramolecular geometry, and the librational and vibrational frequencies is discussed.

\section{MD Simulations}

The interactions between two methanol molecules are described by a recently developed set of potentials based on a flexible three site model consisting of an oxygen atom, a hydrogen atom and the methyl group as a whole [5]. All three sites bear partial charges. The total potential consists of an intra- and an intermolecular part:

$$
V\left(r_{\alpha \beta}, \varrho_{i}\right)=V_{\text {intra }}\left(\varrho_{i}\right)+V_{\text {inter }}\left(r_{\alpha \beta}\right),
$$

where the $\varrho_{i}$ are internal coordinates and the $r_{\alpha \beta}$ denote distances between sites. The intermolecular part of the potential can be separated into a Coulom- 
bic and a non-Coulombic term:

$$
V_{\text {inter }}\left(r_{\alpha \beta}\right)=q_{\alpha} q_{\beta} e^{2} / r_{\alpha \beta}+V_{\alpha \beta}^{\prime}\left(r_{\alpha \beta}\right),
$$

where the charges assigned to the different sites are $q_{\mathrm{O}}=-0.6|e|, q_{\mathrm{H}}=0.35|e|$ and $q_{\mathrm{CH}_{3}}=0.25|e|$. Further details of the potentials are given in [5], where it is also demonstrated by an MD simulation with 200 methanol molecules in the basic cube that this set of potentials is able to describe many structural and dynamical properties of liquid methanol quantitatively.

The effect of polarity in the nearest neighbor structure of methanol was investigated by scaling of the charges: $q_{\alpha}^{\prime}=\lambda q_{\alpha}$, where the $q_{\alpha}$ are the charges on $\mathrm{O}$, $\mathrm{H}$ and $\mathrm{CH}_{3}$ as given above. Three simulations were performed with $\lambda=1,0.75$ and 0.25 . In each simulation the basic cube contained again 200 molecules and extended after complete equilibration over 5000 time steps equivalent to a total elapsed time of about $1.5 \mathrm{ps}$ with an average temperature of $295 \mathrm{~K}$ without rescaling. The Ewald method was used for the long range Coulombic interactions and the shifted force potential method for the non-Coulombic interactions.

\section{Results and Discussion}

\section{A) Radial distribution and $X$-ray structure functions}

The three site-site RDFs where the $\mathrm{CH}_{3}$-group is involved do not change significantly with the polarity. Therefore they are not depicted here. (They are shown in [5] for full charges.) This result is in good agreement with the outcome of the RISM calculations by Pettitt and Rossky [2].

The $\mathrm{O}-\mathrm{O}, \mathrm{O}-\mathrm{H}$, and $\mathrm{H}-\mathrm{H}$ RDFs are shown in Fig. 1 for the three different scaling parameters $\lambda=1$, 0,75 , and 0.25 . Characteristic values are given in Table 1. As these three RDFs are mainly determined by hydrogen bonding, strong changes with polarity are not unexpected. With decreasing polarity the heights of the first peaks decrease strongly, the nearest neighbor distances increase and the minima are filled up by contributions from non-hydrogen bonded pairs. Also the number of nearest neighbors becomes significantly smaller if $n(r)$ is taken in each case at the minimum of the RDF for $\lambda=1$ (Table 1).

The changes in $g_{\mathrm{OH}}(r)$ and $g_{\mathrm{HH}}(r)$ with polarity agree qualitatively with the results of the RISM calculations of Pettitt and Rossky [2], but there is a discrepancy as far as $g_{\mathrm{OO}}(r)$ is concerned. This discrepancy in $g_{\mathrm{OO}}(r)$ leads necessarily to different conclusions about
Table 1. Characteristic values of the oxygen-oxygen, oxygenhydrogen, and hydrogen-hydrogen radial distribution functions of liquid methanol from MD simulations with three different polarities.

\begin{tabular}{llllll}
\hline$x y$ & $\lambda$ & $r_{\max }[\AA]$ & $g_{x y}\left(r_{\max }\right)$ & $r_{\min }[\AA]$ & $n\left(r_{\min }\right)$ \\
\hline OO & 1 & 2.85 & 3.3 & 3.41 & 2.0 \\
& 0.75 & 2.94 & 2.5 & & 1.6 \\
& 0.25 & 3.03 & 1.1 & & 0.9 \\
OH & 1 & 1.95 & 3.4 & 2.65 & 2.0 \\
& 0.75 & 2.02 & 2.4 & & 1.8 \\
& 0.25 & 2.13 & 0.9 & & 1.4 \\
$\mathrm{HH}$ & 1 & 2.45 & 3.0 & 3.31 & 2.3 \\
& 0.75 & 2.55 & 2.0 & & 2.0 \\
& 0.25 & - & 1.0 & & 1.4 \\
\hline
\end{tabular}

the effect of polarity on the structure of methanol. From the only moderate changes in the $\mathrm{O}-\mathrm{O}$ RDF found by Pettitt and Rossky they concluded that the lack of charges mainly results in an unhindered rotation about the $\mathrm{O}-\mathrm{C}$ bond thus influencing $g_{\mathrm{OH}}(r)$ and $g_{\mathrm{HH}}(r)$ while the packing of the molecules remains practically unchanged. In the light of the MD results presented here which show strong changes in $g_{\text {OO }}(r)$ with polarity this conclusion has to be revised in the sense that not only the orientation of the $\mathrm{O}-\mathrm{H}$ bond changes with decreasing polarity but also the relative orientation of neighboring molecules and with it the local packing.

In Fig. 2 the total intermolecular X-ray structure functions are shown for the simulations with $\lambda=1$ and $\lambda=0.25$. There are significant differences between the two curves which are obviously a consequence of the strong changes of $g_{\mathrm{OO}}(r)$ with polarity. Contrary to the conclusion drawn by Pettitt and Rossky [2] from their RISM calculation it has to be concluded that hydrogen bonding contributes considerably to the intermolecular X-ray structure function.

\section{B) Pair interaction energy distribution}

The distributions of the interaction energies between two methanol molecules are presented in Fig. 3 for the three different polarities. The peaks at the negative energy side result from the nearest neighbor interactions. The maxima appear at $-21.3,-13.6$, and $-6.4 \mathrm{~kJ} / \mathrm{mol}$ for the $\lambda$ values $1,0.75$, and 0.25 , respectively. The peak heights decrease with decreasing $\lambda$ as the number of nearest neighbors - defined by $n_{\mathrm{OO}}\left(r_{\min }\right)$ - becomes smaller (Table 1$)$. The energy of $-6,4 \mathrm{~kJ} / \mathrm{mol}$ corresponding to $\lambda=0.25$ results mostly 

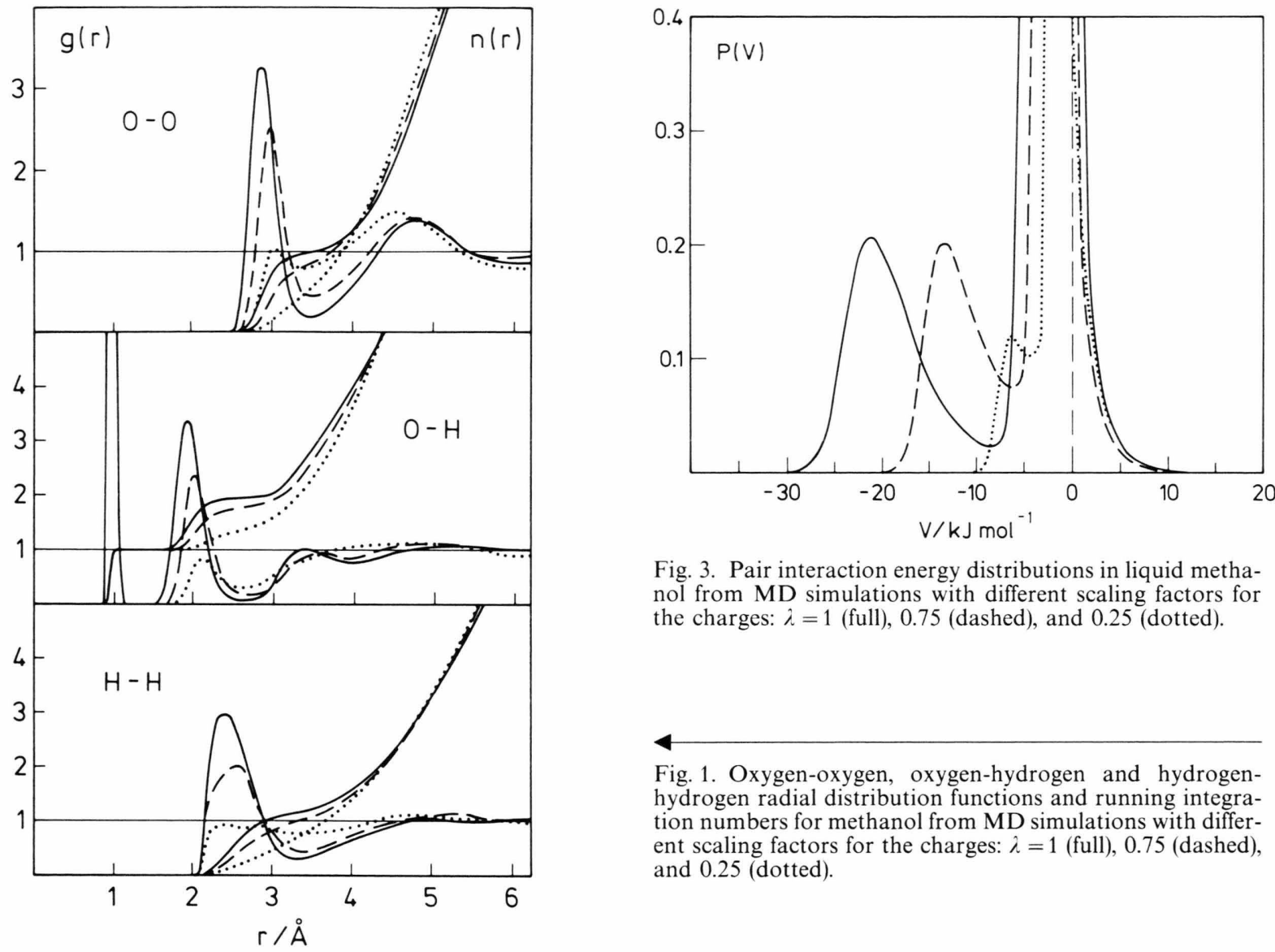

Fig. 3. Pair interaction energy distributions in liquid methanol from MD simulations with different scaling factors for the charges: $\lambda=1$ (full), 0.75 (dashed), and 0.25 (dotted).

Fig. 1. Oxygen-oxygen, oxygen-hydrogen and hydrogenhydrogen radial distribution functions and running integration numbers for methanol from MD simulations with different scaling factors for the charges: $\lambda=1$ (full), 0.75 (dashed), and 0.25 (dotted).

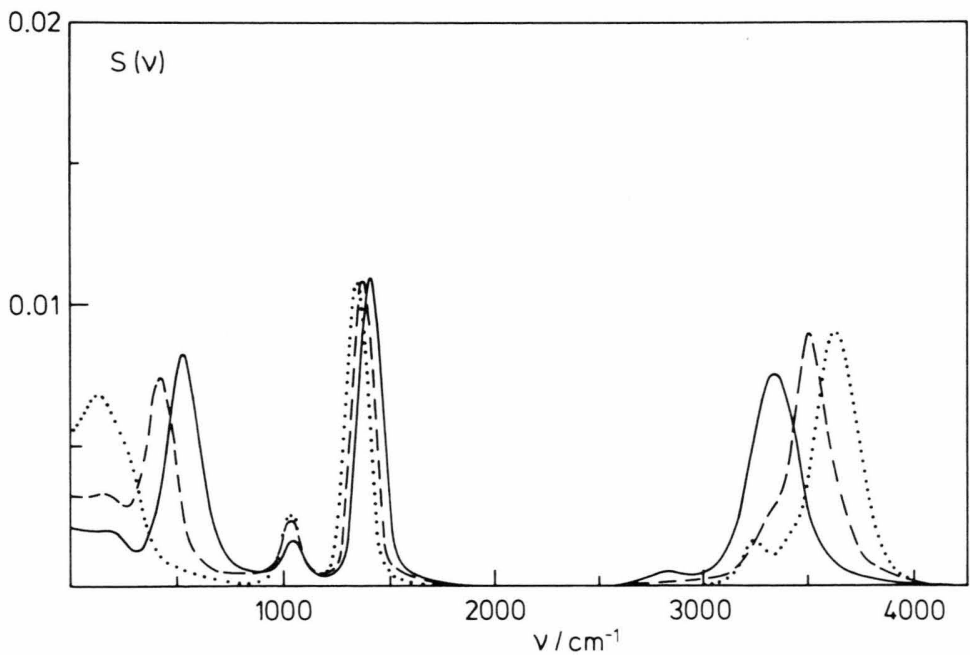

Fig. 2. Total intermolecular X-ray structure functions for methanol from MD simulations with $\lambda=1$ (full) and $\lambda=0.25$

Fig. 4. Power spectra of liquid methanol from MD simulations with different scaling factors for the charges: $\lambda=1$ (full), 0.75 (dashed), and 0.25 (dotted). (dotted). 
from the non-Coulombic term of the intermolecular potential [5]. A further decrease of $\lambda$ would therefore not change this distribution significantly. The nonCoulombic part of the average potential energy changes by about $25 \%$ with the change in polarity. This is a further indication that the local packing depends on the polarity of the methanol molecule.

\section{C) Intramolecular geometry and vibrations}

The intermolecular interactions in the liquid change both the intramolecular geometry and the frequencies of the internal vibrations relative to the gas phase. Therefore, these properties will depend on the polarity. The gas phase values are calculated from a simulation where all intermolecular interactions have been switched off [5].

The average $\mathrm{O}-\mathrm{H}$ bond lengths and the bond angles of the methanol molecules for the three different polarities are compared in Table 2 with the gas phase values. As expected with decreasing $\lambda$ the intramolecular geometry approaches the gas phase one monotonously. The $\mathrm{CH}_{3}-\mathrm{O}$ bond length does not change with polarity in the limits of uncertainty.

The changes of the power spectra with polarity are shown in Figure 4. They have been calculated from the simulations through the velocity autocorrelation functions of the hydrogen atoms. The positions of the band maxima for the different scaling factors $\lambda$ are

[1] B. M. Pettitt and P. J. Rossky, J. Chem. Phys. 77, 1451 (1982).

[2] B. M. Pettitt and P. J. Rossky, J. Chem. Phys. 78, 7296 (1983).

[3] O. Matsuoka, E. Clementi, and M. Yoshimine, J. Chem. Phys. 64, 1351 (1976).

\begin{tabular}{lll}
\hline$\lambda$ & $r_{\mathrm{OH}}$ & $\alpha_{\mathrm{COH}}$ \\
\hline 1 & 0.964 & 107.4 \\
0.75 & 0.957 & 108.0 \\
0.25 & 0.953 & 108.4 \\
gas & 0.945 & 108.5 \\
\hline
\end{tabular}

Table 2. Average $\mathrm{O}-\mathrm{H}$ bond lengths and $\mathrm{C}-\mathrm{O}-\mathrm{H}$ angles of the methanol molecule from MD simulations with three different scaling factors $\lambda$ together with the gas phase values.

Table 3. Frequencies in wavenumbers of the band maxima of the librations and intramolecular vibrations of methanol from MD simulations with three different scaling factors $\lambda$ together with the gas phase values. $\left({ }^{\mathrm{a}}\right.$ frequency of free rotation at $295 \mathrm{~K}$ ).

\begin{tabular}{lclll}
\hline$\lambda$ & $v_{\text {lib. }}$ & $v_{\mathrm{CO}}$ & $v_{\mathrm{COH}}$ & $v_{\mathrm{OH}}$ \\
\hline 1 & 528 & 1055 & 1407 & 3342 \\
0.75 & 426 & 1041 & 1371 & 3506 \\
0.25 & 138 & 1030 & 1344 & 3630 \\
gas & $(120)^{\mathrm{a}}$ & 1030 & 1331 & 3693 \\
\hline
\end{tabular}

compared in Table 3 with corresponding gas phase values. With decreasing $\lambda$ the librational frequencies approach the value for the free rotation of the methanol molecule. The $\mathrm{C}-\mathrm{O}$ stretching frequency does not depend significantly on $\lambda$ in agreement with the unchanged average $\mathrm{CH}_{3}-\mathrm{O}$ distance. The frequency of the bending vibration and the $\mathrm{O}-\mathrm{H}$ stretching frequency depend, as expected, on the strength of the hydrogen bond.

[4] W. L. Jorgensen, J. Amer. Chem. Soc. 103, 335, 341 (1981).

[5] G. Pálinkás, E. Hawlicka, and K. Heinzinger, J. Phys. Chem. 91, 4334 (1987). 